\title{
Self-Adapting Load Balancing for DNS
}

Jörg Jung, Simon Kiertscher, Sebastian Menski, and Bettina Schnor

\author{
University of Potsdam \\ Institute of Computer Science \\ Operating Systems and Distributed Systems
}




\section{Before we start ...}

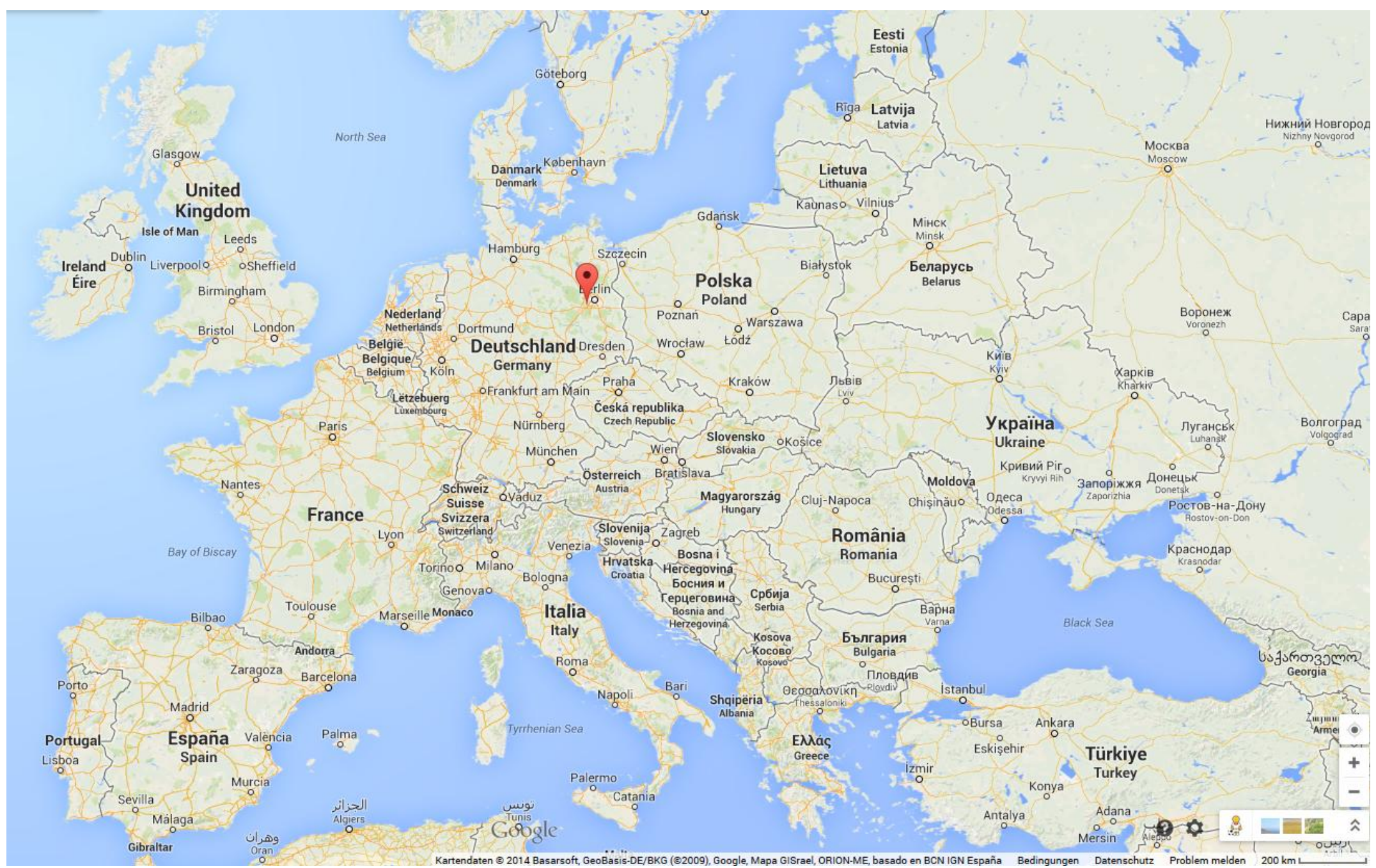




\section{Outline}

1. Introduction

2. Credit based SLB

3. Implementation

4. Measurements and Evaluation

5. Conclusion and Future Work 


\section{Introduction}

\section{Dispatcher based Server Load Balancing (SLB):}

scalable, flexible, and fault tolerance services

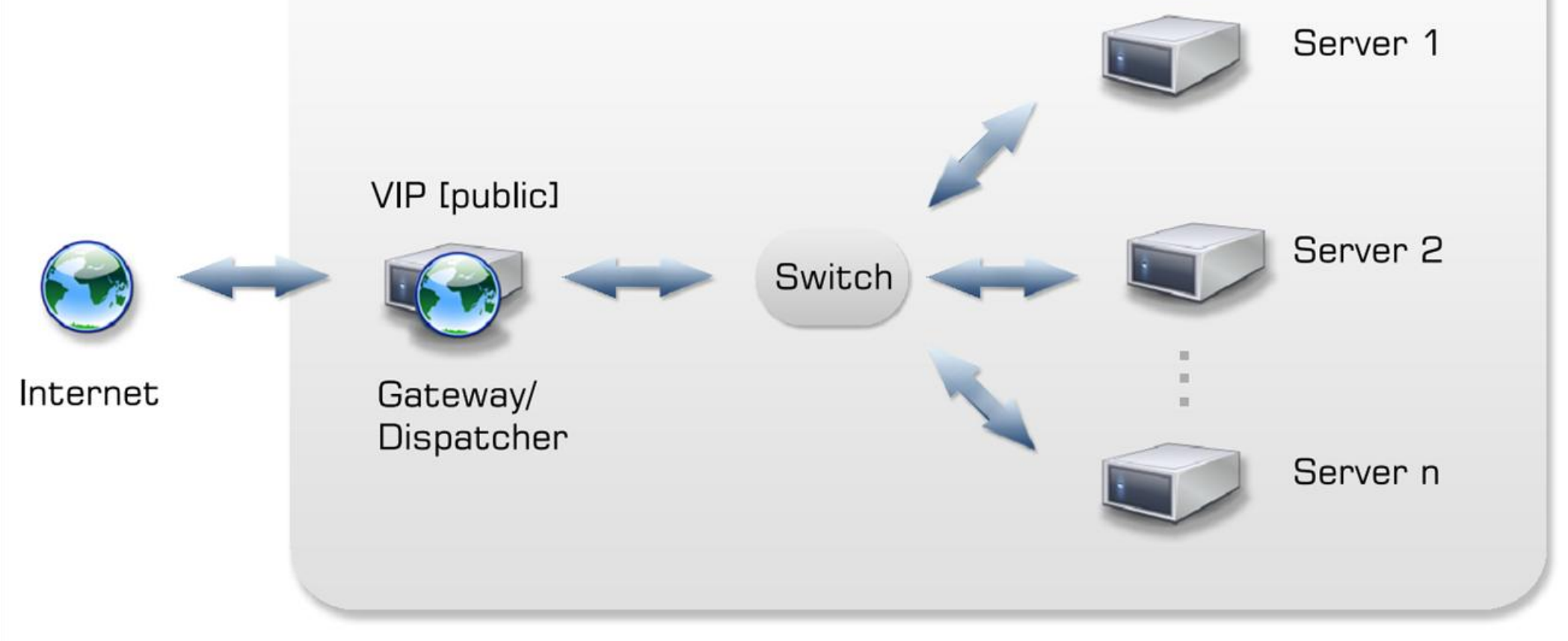




\section{Motivation}

- Sophisticated algorithms are required for heterogeneous workloads and heterogeneous back end servers

- No weights to determine/tune

- Self-adapting credit based SLB for better performance

- Measurements show the advantages of credit based SLB for TCP services like HTTP [Jung et al. 2014]

- contribution:

$\rightarrow$ Efficient implementation for UDP services like DNS required, using a suited credit metric

$\rightarrow$ Measurements to compare traditional and credit based SLB algorithms: WRR vs. DPR 


\section{Credit based SLB}

- introduced in [Jung et al. 2014]:

- Application independent metrics are used to calculate credits

- Back end server push credits to the LB

- Linux environments

- Credits represent the free capacity of the UDP receive queue / TCP backlog queue (in number of UDP requests or TCP connections) 


\section{receive queue vs backlog queue}
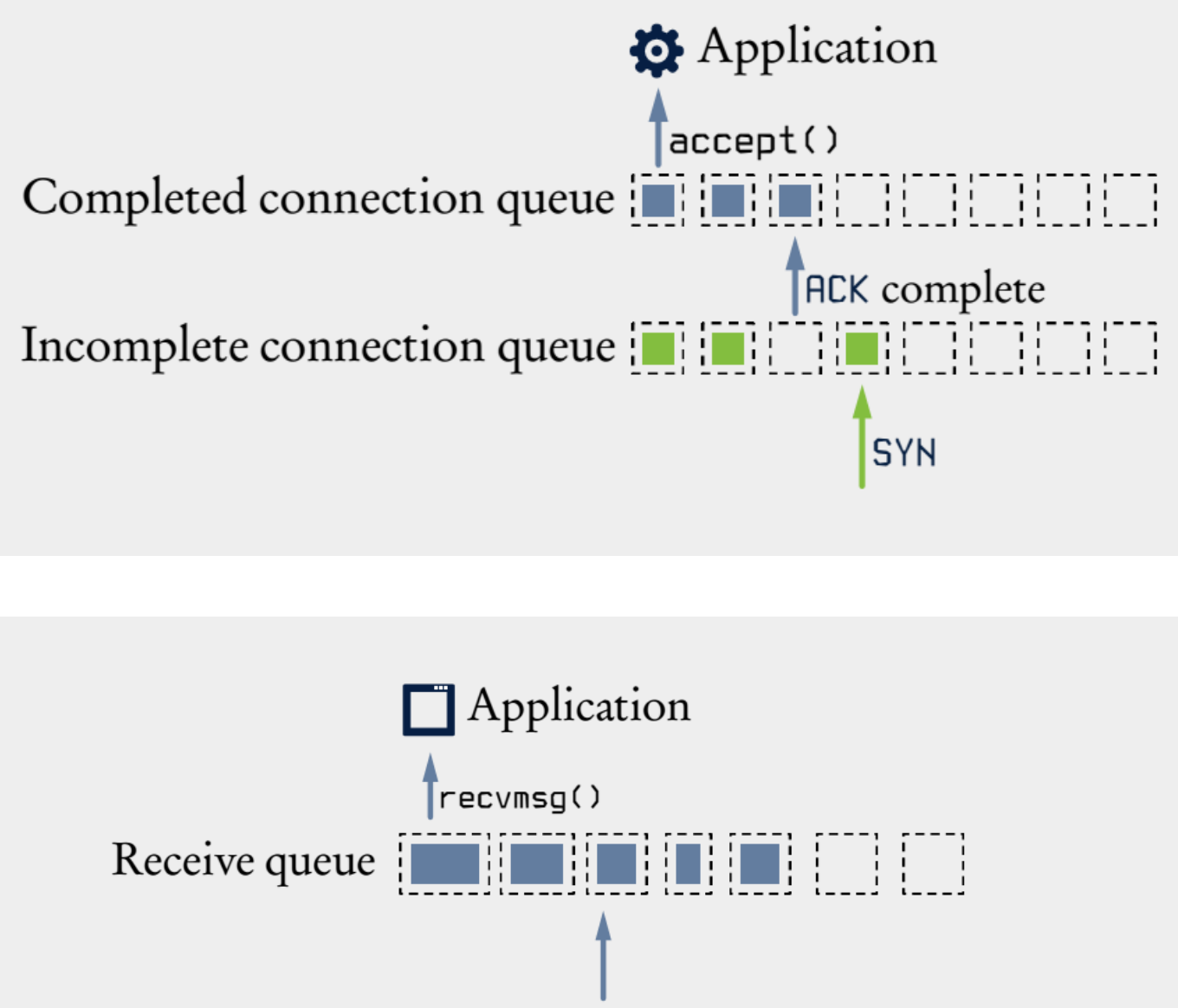


\section{Credit Metric: UDP Receive Queue}

- UDP receive queue as credit metric implicitly reflects the current load on the back end servers:

- A filled receive queue indicates a busy application

- With a full queue the application if fully engaged and might not respond to requests 


\section{Implementation - Overview of salbnet}

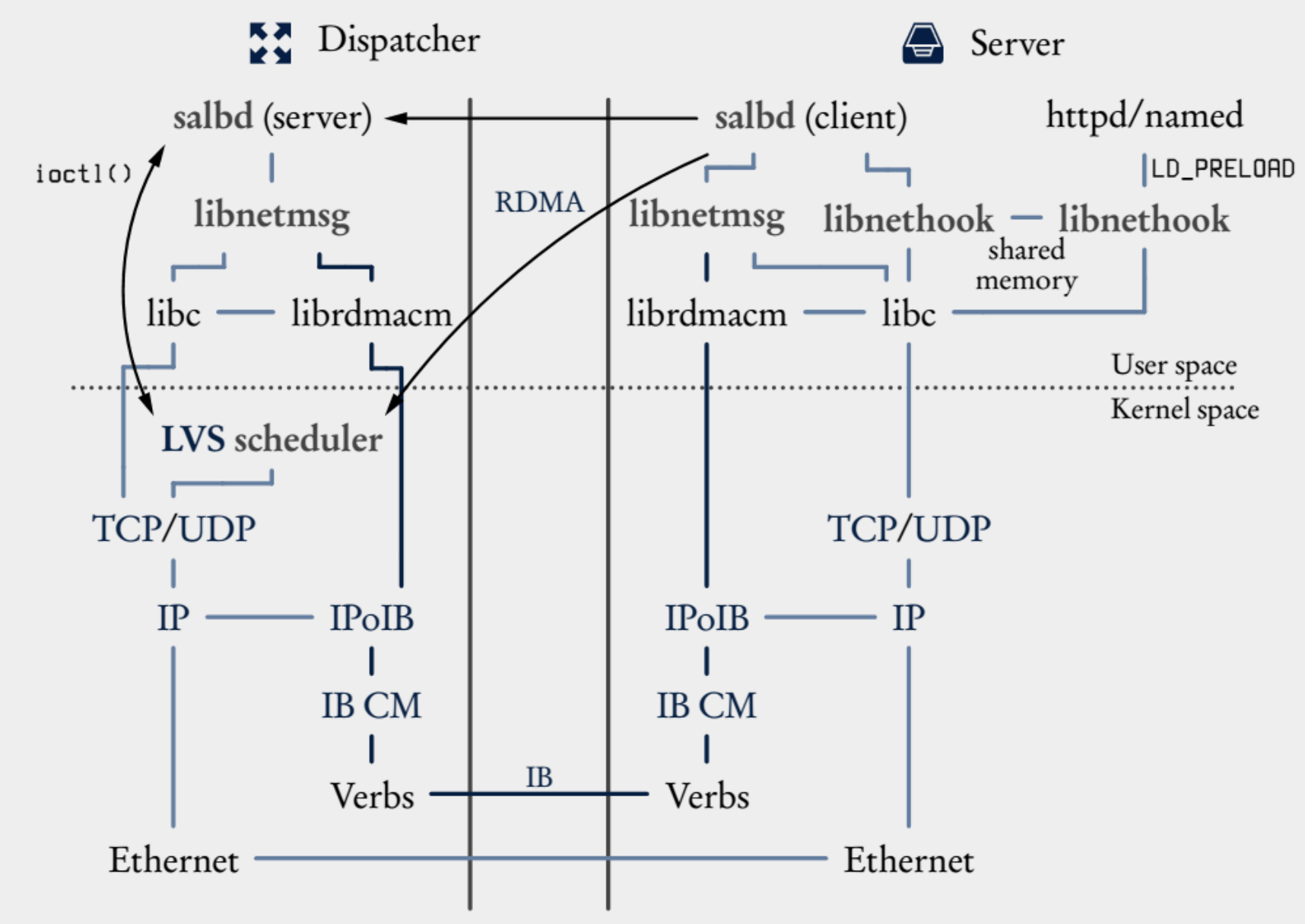




\section{Implementation}

- Using salbnet libnethook for recvmsg() system call interception

$\rightarrow$ get size of the received message (payload)

$\rightarrow$ transparent for UDP applications, like DNS server (BIND)

- UDP packet size and required space in memory needs to be predicted

$\rightarrow$ Find out free capacity

$\rightarrow$ Experimentally determined for Linux kernel

$\rightarrow$ Proof of concept prototype: dependent on specific kernel version 


\section{Measurements and Evaluation}

- Measurements in Internet Service Provider (ISP) like SLB environment using BIND instances

- Heterogeneous hardware and homogenous software versions

- WRR vs. DPR

- 3 heterogeneous back end servers require weights for traditional WRR algorithm

$\rightarrow$ Determined in beforehand measurements [Zinke and Schnor 2013] 


\section{Environment: Setup}

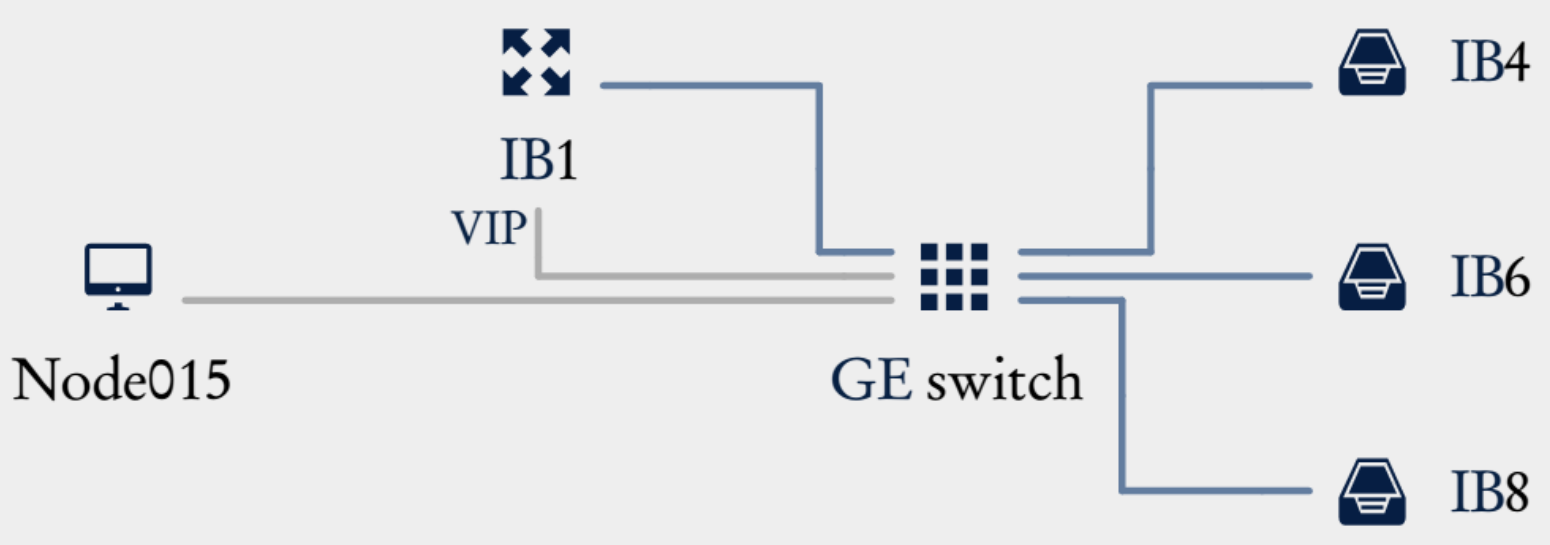




\section{Environment: Hardware}

\begin{tabular}{|l|l|}
\hline Hostname & CPU \\
\hline LVS and IB4 & Dual 1.8 GHz AMD Opteron 244 \\
\hline IB6 & $2.8 \mathrm{GHZ}$ Intel Pentium 4 \\
\hline IB8 & $1.86 \mathrm{GHz}$ Dual Core Intel Xeon 3040 \\
\hline
\end{tabular}

-All machines have 4 GByte memory and GBit links

- Client (load generator) runs with 12 GByte memory 


\section{Benchmark: servload [Zinke et al. 2012]}

- Existing DNS benchmarks like queryperf or DNSPerf and ResPerf are not able to replay queries

- In contrast benchmark servload tries to replay real user sessions

$\rightarrow$ Factor feature replicates real user sessions

$\rightarrow$ Support for DNS protocol added to servload 


\section{Environment: Software}

- 3 BIND DNS servers v9.3.6

- LVS LB with ipvsadmin v1.24

- Client with servload v0.5.1

- OS LB and Servers: CentOS Linux 5.7 with kernel 2.6.18

- OS Client: Debian Linux with kernel 2.6.26

- Monitoring: SNMPv1 requests once a minute from LB (in addition to the credits) 


\section{Workload: Increased DNS Traces}

Number of requests from five minutes of a trace from the 29th September 2011 from DNS servers running

haiti.cs.uni-potsdam.de

\begin{tabular}{|r|r|r|r|r|}
\hline Factor & Requests & Sessions & Mean req/s & Max req/s \\
\hline 1 & 22,594 & 33 & 75.31 & 204 \\
\hline 400 & $9,037,600$ & 13,200 & 30,125 & 81,600 \\
\hline 800 & $18,075,200$ & 26,400 & 60,250 & 163,200 \\
\hline 1,600 & $36,150,400$ & 52,800 & 120,501 & 326,400 \\
\hline
\end{tabular}




\section{Results: (First) Response Time}

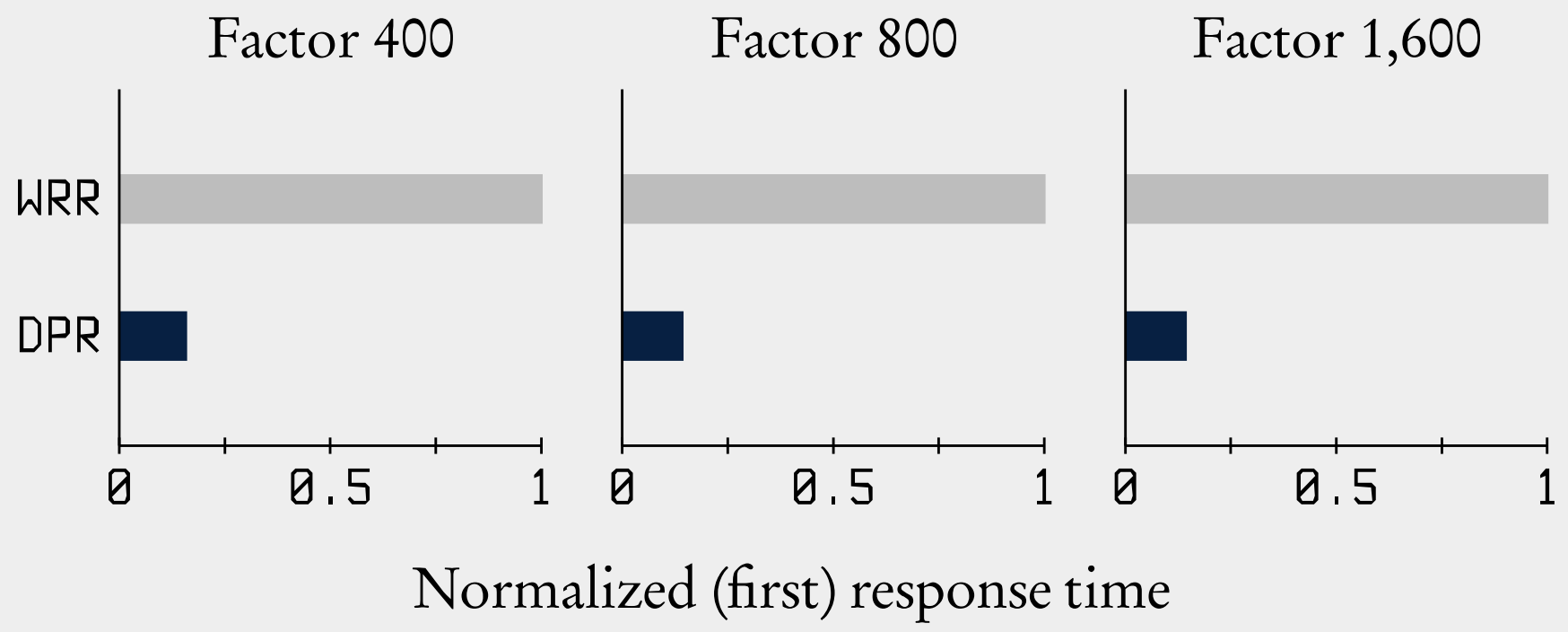

\begin{tabular}{|l|r|r|r|}
\hline Method & F 400 & F 800 & F 1600 \\
\hline WRR & $4.05 \mathrm{~ms}$ & $4.33 \mathrm{~ms}$ & $4.24 \mathrm{~ms}$ \\
\hline DPR & $0.64 \mathrm{~ms}$ & $0.62 \mathrm{~ms}$ & $0.60 \mathrm{~ms}$ \\
\hline
\end{tabular}




\section{Results: (Request) Errors}

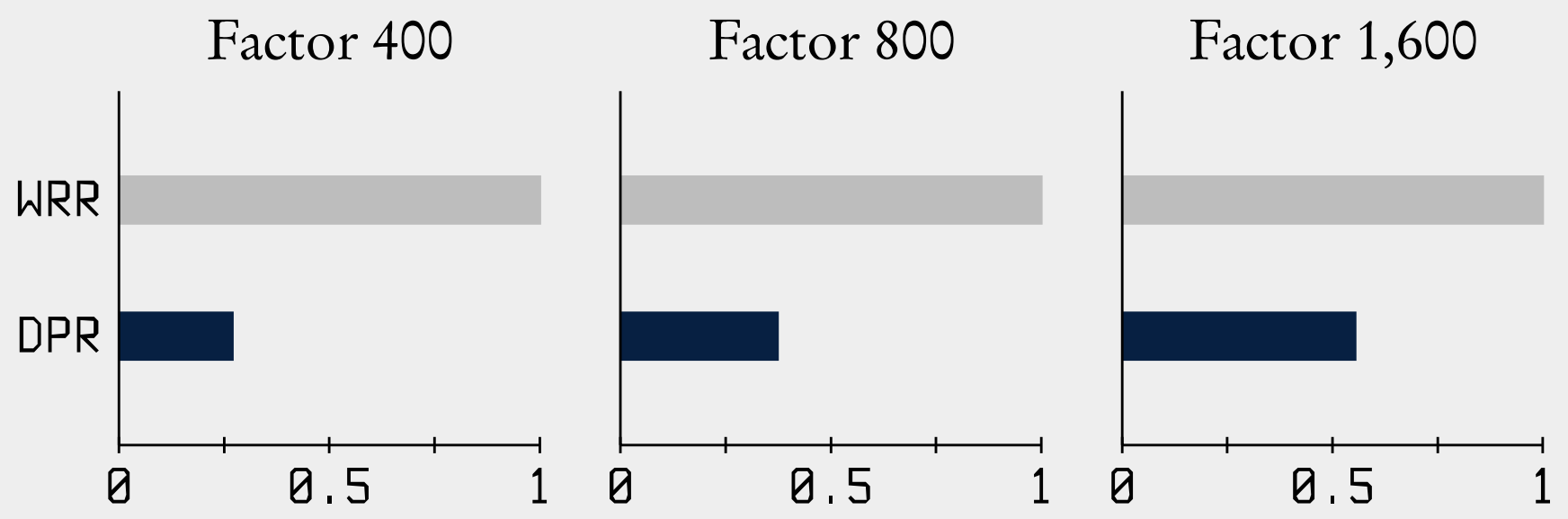

Normalized (request) errors

\begin{tabular}{|l|r|r|r|}
\hline Method & F 400 & F 800 & F 1600 \\
\hline WRR & $1273(0.01 \%)$ & $2038(0.01 \%)$ & $2910(0.01 \%)$ \\
\hline DPR & $343(0.00 \%)$ & $759(0.00 \%)$ & $1612(0.00 \%)$ \\
\hline
\end{tabular}




\section{Conclusions and Future Work}

- Credit based SLB extended to support UDP services

- servload benchmark extended to work with DNS

- Previous TCP (HTTP) measurements are confirmed

- DPR outperforms traditional WRR with already tuned weights

- by $15 \%$ (FRT) and $25 \%-50 \%$ (error rate)

- salbnet scales

- Next: transition from proof concept to mature solution 


\section{Thank you for your attention! Any questions?}

Contact:

jozinke@cs.uni-potsdam.de

www.cs.uni-potsdam.de 


\section{References}

- queryperf is part of the BIND distribution available from: [ISC 2014] ISC. BIND | Internet Systems Consortium, May 2014. URL http:// www.isc.org/downloads/bind/. Accessed May 2014.

- DNSPerf and ResPerf are available from: [Nominum 2014] Nominum.

Measurement Tools | Nominum, February 2014. URL http://www.nominum.com/support/measurement-tools/. Accessed May 2014.

- [Zinke et al. 2012] Jörg Zinke, Jan Habenschuß, and Bettina Schnor. servload: Generating Representative Workloads for Web Server Benchmarking. In Proceedings of the 15th International Symposium on Performance Evaluation of Computer and Telecommunication Systems, pp. 82-89. SPECTS 2012. IEEE Communications Society. Society for Modeling \& Simulation International (SCS), Genoa, Italy, July 2012.

- [Zinke and Schnor 2013] Jörg Zinke and Bettina Schnor. The Impact of Weights on the Performance of Server Load Balancing Systems. In Proceedings of the 16th Internation- al Symposium on Performance Evaluation of Computer and Telecommunication Systems, pp. 541-548. SPECTS 2013. IEEE Communications Society. Society for Modeling \& Simulation International (SCS), Toronto, Canada, July 2013.

- [Jung et al. 2014] Jörg Jung, Bettina Schnor, and Sebastian Menski. salbnet: A Self-Adapting Load Balancing Network. In Proceedings of the 12th IASTED International Conference on Parallel and Distributed Computing and Networks, pp. 249-257. PDCN 2014. ACTA Press. IASTED, Innsbruck, Austria, February 2014. 